\title{
Toward developing a simulated workplace laboratory for adults with high functioning autism spectrum disorders
}

\author{
Ronna Fried $^{1,2}$, Gagan Joshi ${ }^{1,2}$, Amelia Kotte ${ }^{1}$, Elana Kagan ${ }^{1}$, Joseph Biederman ${ }^{1,2}$ \\ ${ }^{1}$ Pediatric Psychopharmacology Program, Massachusetts General Hospital, Boston, USA \\ ${ }^{2}$ Department of Psychiatry, Harvard Medical School, Boston, USA \\ Email: rfried@partners.org
}

Received 4 April 2013; revised 5 May 2013; accepted 14 May 2013

Copyright (c) 2013 Ronna Fried et al. This is an open access article distributed under the Creative Commons Attribution License, which permits unrestricted use, distribution, and reproduction in any medium, provided the original work is properly cited.

\begin{abstract}
Objective: Adults with high functioning autism spectrum disorders (HF-ASD) often experience difficulty in sustaining employment, yet little is known about workplace deficits in this population. This study aimed to assess the workplace performance of individuals with HF-ASDs in a previously validated laboratory workplace simulation environment. Method: Seven subjects of both sexes, ages 18 - 21 with a clinical diagnosis of ASD were brought in for a 10hour workplace simulation, with a series of structured and unstructured tasks designed to elicit DSMIV Attention Deficit and Hyperactivity Disorder (ADHD) symptoms and to provide objective measures of workplace performance. Results: HF-ASD participants performed significantly worse than Controls on several tasks throughout the day, particularly in the afternoon. Although HF-ASD subjects did not report elevated symptoms of ADHD, they completed significantly less of the unstructured tasks than Controls. Conclusion: These findings suggest that unstructured work activities and a lack of self-awareness are two primary areas of workplace deficits for individuals with HF-ASD.
\end{abstract}

Keywords: Workplace; Employment; Autism Spectrum Disorder; Self-Report

\section{INTRODUCTION}

Autism Spectrum Disorders (ASDs) comprise a group of developmental disorders characterized by variable presentations of difficulty in socialization, communication, and behavior estimated to affect at least $1 \%$ of individu-

*This study was supported by funds from the Alan and Lorraine Bressler Clinical and Research Program for Autism Spectrum Disorders at Massachusetts General Hospital. als in the general population [1,2]. There is increased recognition of ASD in intellectually capable populations (high functioning autism spectrum disorder, or HF-ASD) [3].

Despite adequate intellectual and language abilities, adults with HF-ASD often experience considerable challenges in securing and sustaining competitive employment [4-8]. The economic cost due to loss of productivity in working age adults with HF-ASD is estimated to range from $\$ 39,000$ to $\$ 130,000$ per annum [9], suggesting that the economic impact of workplace difficulties in individuals with HF-ASD can be enormous [10-12]. Despite these data, there has been very limited research on the nature of workplace deficits in individuals with HFASD.

It is critical to better understand deficits in functioning within the context of the work environment in order to develop appropriate intervention strategies. As obtaining information from employers or observing adults with HF-ASD in the workplace is not practical, and, by nature of the disorder, adults with ASD are not good reporters of their deficits, there is a pressing need to develop novel approaches and examine functioning in the work environment. One approach to nonintrusive gain insight into the nature of workplace deficits in individuals with HFASD is through the use of a simulated laboratory workplace environment. Our group recently validated a full day laboratory workplace simulation for individuals with Attention Deficit and Hyperactivity Disorder (ADHD) and found robust differences in a number of key areas, including differences in self-report of ADHD symptoms in participants with ADHD and Controls during the simulated workday [13]. Because a large number of individuals with HF-ASD also suffer from ADHD [14], we posited that a similar experimental laboratory workplace paradigm could be informative to assess workplace deficits in individuals with HF-ASD.

The main aim of this study was to assess the work- 
place performance of individuals with HF-ASDs in a laboratory workplace simulation environment. Due to the high comorbidity with ADHD, in conjunction with their propensity to have deficits in self-awareness, we hypothesized that adults with HF-ASD would exhibit impairment on tasks requiring sustained attention, would self report more symptoms of ADHD throughout the day, and would exhibit symptoms of ADHD that would be observable to objective raters. To the best of our knowledge, this represents the first systematic effort to date to assess workplace deficits in individuals with HF-ASD using a laboratory workplace simulation.

\section{METHODS}

ASD subjects were youth, ages 18 - 21 years of age referred to a specialized program for the treatment of ASDs at a university-affiliated hospital. All ASD subjects underwent a comprehensive psychiatric evaluation by an expert clinician (GJ) that included a detailed assessment with the patient and the parent and diagnosis based on DSM-IV criteria. Full description of psychiatric assessments are found in previous work [15] but briefly reported here. Based on this expert interview, all ASD subjects met Diagnostic and Statistical Manual of Mental Disorders-Fourth Edition (DSM-IV) diagnostic criteria for autistic disorder, Asperger's disorder, or PDD-NOS. Control subjects were age and sex matched adults recruited from advertising in the local media. Interested participants underwent a telephone-screening questionnaire that asked about symptoms of ADHD, as well as study inclusion and exclusion criteria. An expert clinician completed a comprehensive psychiatric evaluation, which included a detailed assessment of ADHD based on DSM-IV criteria, and ruled out the diagnosis of ADHD.

Neurocognitive measures were administered by trained psychometricians who were blind to diagnostic/ascertainment status and who received ongoing supervision by a licensed clinical neuropsychologist. The neuropsychological battery was administered in a fixed order: 1) Wide Range Achievement Test (WRAT) Math, 2) Wechsler Abbreviated Scale of Intelligence (WASI) Vocabulary, 3) Wechsler Adult Intelligence Scale-III (WAIS-III) Digit Span, 4) Delis-Kaplan Executive Function System (D-KEFS) Color-Word Interference Test, 5) WAIS-III Digit/Symbol Coding, 6) WASI Matrix, 7) WAIS-III Letter Number Sequencing, 8) WAIS-III Symbol Search, 9) Test of Word Reading Efficiency (TOWRE), 10) WAIS-III Oral Arithmetic, and 11) D-KEFS Trail Making [16-18]. In addition to these subtests, modules from the Cambridge Neuropsychological Test Automated Battery (CANTAB) [19] were administered: Verbal Recognition Memory (VRM); Intra-Extra Dimensional Set Shift (IED); Spatial Working Memory (SWM); Stockings of Cambridge (SOC); Reaction Time (RTI); Rapid Visual
Information Processing (RVP); and Affective Go/No-Go (AGN).

\subsection{Simulated Workplace Laboratory}

Detailed methodology of the workplace simulation has been previously reported [13]. Briefly, the laboratory workplace was designed to simulate a full workday, with participants arriving at 8:30 a.m. and departing at 6:30 p.m. Within the 10-hour day, there were three periods each of structured and unstructured time of 90 minutes each, and a lunch hour. During the structured periods, participants worked on specific tasks, each for a predetermined length of time. During the unstructured periods, participants were given a packet containing a variety of tasks and distracters, with the instruction that the packets were due by the end of the day. A detailed instruction manual was used with pre-recorded directions for tasks to ensure consistency. Tasks were designed to elicit DSM-IV symptoms of ADHD and to provide objective measures of work performance. During tasks, participants were rated blindly by trained observers using strictly defined measures of behavioral observations, and participants completed self-evaluations measuring symptoms associated with ADHD.

During the structured periods of the workplace simulation day, participants were asked to complete seven specific tasks within 1 hour and 30 minutes.

Video: Participants viewed a 25-minute educational video and were asked to complete six questions of comparable difficulty about each section of the video.

Math: The 5 minute math fluency test started with simple addition and subtraction problems and moved on to include multiplication and division.

Lecture: The lecture task assessed the ability to listen to a lecture, take notes, and use the notes to answer questions. Participants were instructed to listen to a recording while taking notes on content. Later in the day, these notes were returned and participants were instructed to answer questions regarding the lecture using their notes.

Reading comprehension: This task was designed to assess silent reading and reading comprehension. Texts were chosen based on similarity to reading that might be required in the workplace; they were lengthy, but did not contain obscure vocabulary. Each passage was followed by a series of questions, with the text available to the participants for referral. This reading inventory was also utilized in the unstructured period.

Editing: Participants were presented with brief typed documents containing intentional spelling, grammar, formatting, and referencing mistakes, and asked to circle mistakes in red pen.

During unstructured periods, participants were asked to work on a packet containing a variety of tasks and 
distracters. The packet included a list of tasks that were required. Participants were expected to monitor their time and meet the deadline for each task in the packet without guidance.

Forms and questionnaires: Participants were asked to complete a number of forms during each unstructured period, including a variety of self-report questionnaires and a request for parking validation.

Logic: Logic tasks were administered in assigned groups of four people to assess teamwork and coworker perceptions of behavior. Following completion of the task, a peer report form was distributed to all group members and they were asked to report on the behaviors of each group member during the task.

Writing: We administered writing tasks of the Wechsler Individual Achievement Test (WIAT), which is written for adults. One writing task was from the WIAT-I, and two from the WIAT-II.

Computer tasks: Participants were given detailed instructions about how to log into Microsoft Outlook, and about sending an exact message to four different fabricated email addresses.

Product orders: Participants were given three catalogs containing information about various products and order forms. Participants were asked to place orders based on specifications from the catalogs, using order forms.

Distracter tasks: The work simulation packets also contained items intended to distract the participants, including Sudoku and crossword puzzles, reading materials, and a gift certificate to the hospital gift store. Participants were told explicitly that these tasks were optional, and might be used during down time. Participants were free to take breaks or leave the room as they pleased during unstructured time.

Throughout the day, raters assessed the observable behaviors of the participants, including inattention, restlessness, and disruption. Raters received extensive training on specific criteria, as has been described previously [13]. In addition to observer ratings, participants were asked to complete a self-report rating scale measuring their internal experience after each task. A peer rating scale captured participants' inattention for the logic task.

We used the observer-rating inventory (ORI) rating system as a way of measuring the frequency of hyperactive or inattentive actions and then multiplied it by the intensity of those actions, which was also determined from the ORI system. This frequency $\times$ intensity score gives us a better picture of how much more inattentive or hyperactive one participant was in comparison to another.

\subsection{Statistical Analysis}

Because of the large number of potential comparisons possible, we took a sequential approach to significant testing. First we conducted for each outcome measure of inattention and hyperactivity a separate overall 3 (Group) $\times 15$ (Tasks) Factorial MANOVA for each of the tasks across each of the three time points. If this overall MANOVA was significant at $p<0.05$, we then conducted separate one-way ANOVA for each task at each time point for each dependent variable. To test for diminishing performance across time points within the HFASD group, we conducted a one-way ANOVA for each task across time points. Where this omnibus ANOVA was significant at $p<0.05$, we conducted post-hoc LSD tests for all pair-wise comparisons of the three groups as well as of the three time points for the within HF-ASD group comparisons. We considered significant all posthoc tests at $p<0.05$. We used this sequential approach to protect against Type I error rather than the more conservative Bonferroni correction because of the small sample size of the HF-ASD group.

\section{RESULTS}

Seven individuals diagnosed with Autism Spectrum Disorder took part in this pilot study. Participants with HF-ASD were younger than the ADHD and Control groups, but participants with HF-ASD were from similar SES backgrounds (see Table 1).

As shown in Table 2, in the morning tasks HF-ASD participants performed significantly worse than Controls on the Math Fluency Task (Controls $=133.4 \pm$ 22.7, HF-ASD $=104.57 \pm 30.5, F=5.74, p=0.004, F$ $=5.74, p<0.004)$. There were no other significant morning differences between HF-ASD participants and either Controls or ADHD participants on any of the other six tasks.

In the early afternoon tasks, HF-ASD performed worse than Controls on the timed math fluency task (Controls $=118.5 \pm 33.9$, HF-ASD $=91.4 \pm 35.48, F=$ 3.87, $p=0.023$ ) and the Editing Grammar task (Controls $=3.7 \pm 1.4$, HF-ASD $=2.57 \pm 1.61$, ADHD $=3.1$ $\pm 1.6, F=3.85, p=0.024$ ).

In the late afternoon tasks, the HF-ASD participants performed significantly worse than Controls in the Ed-

Table 1. Demographics.

\begin{tabular}{cccc}
\hline & ADHD $(N=56)$ & Controls $(N=63)$ & ASD $(N=7)$ \\
\hline & Mean \pm SD or & Mean \pm SD or & Mean \pm SD \\
& $N(\%)$ & $N(\%)$ & or $N(\%)$ \\
Age & $28.3 \pm 8.5$ & $30.8 \pm 10.2$ & $19 \pm 1.2$ \\
Sex & $29(54)$ & $25(41)$ & $6(85)$ \\
$\begin{array}{c}\text { Socioeconomic } \\
\text { Status }\end{array}$ & $2.2 \pm 1.2$ & $2.2 \pm 0.9$ & $2.0 \pm 1.2$ \\
$\begin{array}{c}\text { General Assessment } \\
\text { of Functioning }\end{array}$ & $61.0 \pm 4.8$ & $70.5 \pm 2.2$ & $52.9 \pm 3.4$ \\
\hline
\end{tabular}


Table 2. Task scores.

\begin{tabular}{|c|c|c|c|c|c|}
\hline & $\operatorname{ADHD}(N=56)$ & Control $(N=63)$ & $\operatorname{ASD}(N=7)$ & & \\
\hline & & Morning & & $\boldsymbol{F}$ & $p$-value \\
\hline \multicolumn{6}{|l|}{ Structure Tasks } \\
\hline Math Fluency & $122.1 \pm 27.3$ & $133.4 \pm 22.7$ & $104.57 \pm 30.50$ & 5.74 & 0.004 \\
\hline Lecture & $3.8 \pm 1.1$ & $3.7 \pm 1.2$ & $2.85 \pm 1.46$ & 1.98 & 0.143 \\
\hline Reading Implicit & $3.0 \pm 1.2$ & $3.2 \pm 1.0$ & $3.57 \pm 0.53$ & 1 & 0.3699 \\
\hline Reading Explicit & $3.75 \pm 1.1$ & $4.1 \pm 0.9$ & $3.71 \pm 1.11$ & 0.68 & 0.5065 \\
\hline Editing Spelling & $3.4 \pm 1.1$ & $3.6 \pm 1.1$ & $3.28 \pm 0.75$ & 0.66 & 0.5168 \\
\hline Editing Punctuation & $2.6 \pm 1.3$ & $3.2 \pm 1.3$ & $2.14 \pm 1.57$ & 4.19 & 0.0175 \\
\hline \multirow[t]{3}{*}{ Editing Grammar } & $3.6 \pm 1.4$ & $3.9 \pm 1.1$ & $3 \pm 1.63$ & 1.78 & 0.1733 \\
\hline & ADHD $(N=56)$ & Control $(N=63)$ & $\operatorname{ASD}(N=7)$ & & \\
\hline & & Early Afternoon & & $\boldsymbol{F}$ & $p$-value \\
\hline \multicolumn{6}{|l|}{ Structure Tasks } \\
\hline Math Fluency & $103.8 \pm 33.8$ & $118.5 \pm 33.9$ & $91.43 \pm 35.48$ & 3.87 & 0.023 \\
\hline Lecture & $3.6 \pm 1.2$ & $4.0 \pm 0.9$ & $3.85 \pm 0.89$ & 2.53 & 0.0835 \\
\hline Reading Implicit & $4.3 \pm 0.9$ & $4.2 \pm 0.9$ & $3.71 \pm 0.75$ & 1.13 & 0.3271 \\
\hline Reading Explicit & $3.8 \pm 1.2$ & $3.9 \pm 1.0$ & $2.85 \pm 1.77$ & 0.43 & 0.6519 \\
\hline Editing Spelling & $2.9 \pm 1.3$ & $2.9 \pm 1.2$ & $2.28 \pm 1.60$ & 0.8 & 0.4502 \\
\hline Editing Punctuation & $2.9 \pm 1.2$ & $3.5 \pm 1.2$ & $2.85 \pm 1.46$ & 3.41 & 0.0361 \\
\hline \multirow[t]{3}{*}{ Editing Grammar } & $3.1 \pm 16$ & $3.7 \pm 1.4$ & $2.57 \pm 1.61$ & 3.85 & 0.024 \\
\hline & $\operatorname{ADHD}(N=56)$ & Control $(N=63)$ & $\operatorname{ASD}(N=7)$ & & \\
\hline & & Late Afternoon & & $\mathbf{F}$ & $p$-value \\
\hline \multicolumn{6}{|l|}{ Structure Tasks } \\
\hline Math Fluency & $132.9 \pm 24.8$ & $146.1 \pm 19.5$ & $108.29 \pm 34.97^{*}$ & 11.24 & $<0.001$ \\
\hline Lecture & $4.4 \pm 0.8$ & $4.3 \pm 0.8$ & $3.42 \pm 1.4^{*}$ & 4.05 & 0.02 \\
\hline Reading Implicit & $2.8 \pm 1.2$ & $3.0 \pm 1.2$ & $2.14 \pm 1.35$ & 1.77 & 0.1751 \\
\hline Reading Explicit & $3.6 \pm 1.3$ & $3.9 \pm 1.1$ & $2.29 \pm 1.60$ & 2.02 & 0.1383 \\
\hline Editing Spelling & $2.3 \pm 1.2$ & $3.0 \pm 1.3$ & $1.57 \pm 1.4$ & 7.26 & 0.0011 \\
\hline Editing Punctuation & $3.1 \pm 1.3$ & $3.6 \pm 1.3$ & $2 \pm 1.41^{*}$ & 5.48 & 0.0053 \\
\hline Editing Grammar & $3.3 \pm 1.5$ & $4.2 \pm 1.1$ & $3 \pm 1.53$ & 8.42 & $<0.001$ \\
\hline
\end{tabular}

Underlined text was statistically significant $(p<0.05)$ vs. Controls during that time period. ${ }^{*}$ Statistically significant vs. ADHD during that time period.

iting Spelling (Controls $=3.0 \pm 1.3$, HF-ASD $=1.57 \pm$ 1.4 , ADHD $=2.3 \pm 1.2, F=7.26, p=0.001)$ and Editing Grammar tasks (Controls $=4.2 \pm 1.1$, HF-ASD $=3 \pm$ 1.53 , ADHD $=3.3 \pm 1.5, F=8.42, p<0.001)$. Additionally, HF-ASD participants performed significantly worse than Controls and ADHD participants in the following tasks: Math Fluency (Controls = $146.1 \pm 19.5$, HF-ASD $=108.29 \pm 34.97$, ADHD $=132.9 \pm 24.8, F=$ $11.24, p<0.001$ ), Lecture (Controls $=4.3 \pm 0.8$, HF$\mathrm{ASD}=3.42 \pm 1.4, \mathrm{ADHD}=4.4 \pm 0.8, F=4.05, p<0=$ 0.02), and Editing Punctuation (Controls $=3.6 \pm 1.3$, $\mathrm{HF}-\mathrm{ASD}=2 \pm 1.41, \mathrm{ADHD}=3.1 \pm 1.3, F=5.48, p=$ 0.005).

As shown in Table 3, ASD participants demonstrated worse Implicit Reading performance in the late after- noon than in the early afternoon and in the morning ( $F$ $=5.7, p=0.004)$. No other statistically significant differences were observed within tasks across the day's span.

The profiles of symptomatic ratings according to participant self-report (Figures 1 and 2) and trained research observers (Figures $\mathbf{3}$ and $\mathbf{4}$ ) are presented for the full day and broken down into segments: morning, afternoon, and late afternoon. Each task administered is presented across the $\mathrm{X}$-axis according to the work simulation day schedule ( $\mathrm{V}=$ video comprehension, $\mathrm{M}$ = math, $\mathrm{L}=$ lecture, $\mathrm{R}=$ reading comprehension, $\mathrm{E}=$ text editing, $\mathrm{U}=$ unstructured time). The factorial MANOVAs produced significant Group Effect and Group $\times$ Task interactions. The results of the follow-up 
Table 3. Time of day task results.

\begin{tabular}{|c|c|c|c|c|c|}
\hline \multicolumn{6}{|c|}{$\operatorname{ASD}(N=7)$} \\
\hline & Morning & $\begin{array}{c}\text { Early } \\
\text { Afternoon }\end{array}$ & $\begin{array}{c}\text { Late } \\
\text { Afternoon }\end{array}$ & $F$ & $p$-value \\
\hline $\begin{array}{l}\text { Math } \\
\text { Fluency }\end{array}$ & \multicolumn{3}{|c|}{$104.57 \pm 30.5091 .43 \pm 35.48108 .29 \pm 34.97$} & 0.5 & 0.83 \\
\hline Lecture & $2.85 \pm 1.46$ & $3.85 \pm 0.89$ & $3.42 \pm 1.4$ & 2.2 & 0.12 \\
\hline $\begin{array}{l}\text { Reading } \\
\text { Implicit }\end{array}$ & $3.57 \pm 0.53$ & $3.71 \pm 0.75$ & $2.14 \pm 1.35^{*}$ & 5.7 & 0.004 \\
\hline $\begin{array}{l}\text { Reading } \\
\text { Explicit }\end{array}$ & $3.71 \pm 1.11$ & $2.85 \pm 1.77$ & $2.29 \pm 1.60$ & 1.7 & 0.19 \\
\hline $\begin{array}{l}\text { Editing } \\
\text { Spelling }\end{array}$ & $3.28 \pm 0.75$ & $2.28 \pm 1.60$ & $1.57 \pm 1.4$ & 2.6 & 0.08 \\
\hline $\begin{array}{l}\text { Editing } \\
\text { Punctuation }\end{array}$ & $2.14 \pm 1.57$ & $2.85 \pm 1.46$ & $2 \pm 1.41$ & 0.5 & 0.74 \\
\hline $\begin{array}{l}\text { Editing } \\
\text { Grammar }\end{array}$ & $3 \pm 1.63$ & $2.57 \pm 1.61$ & $3 \pm 1.53$ & 0.6 & 0.7 \\
\hline
\end{tabular}

Underlined text was statistically significant $(p<0.05)$ vs. the earlier time of day. "Statistically significant vs. morning.

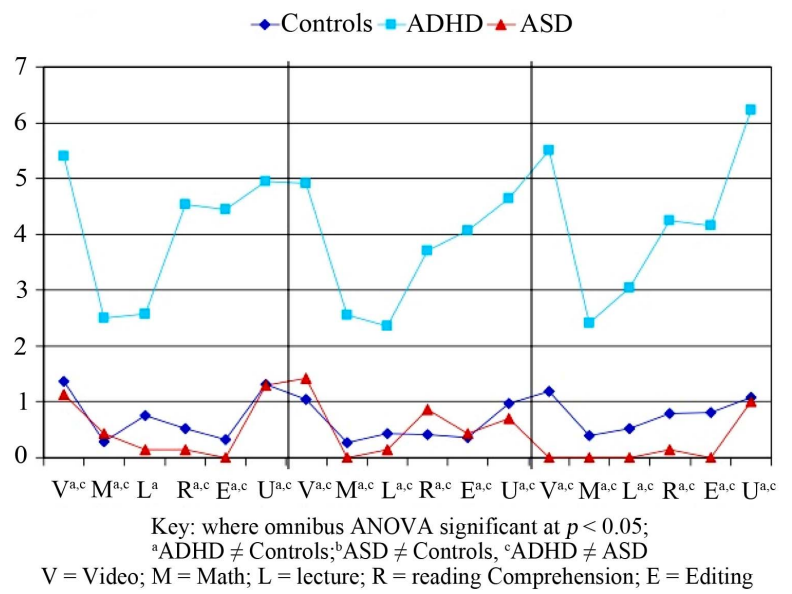

Figure 1. Self rating-hyperactivity.

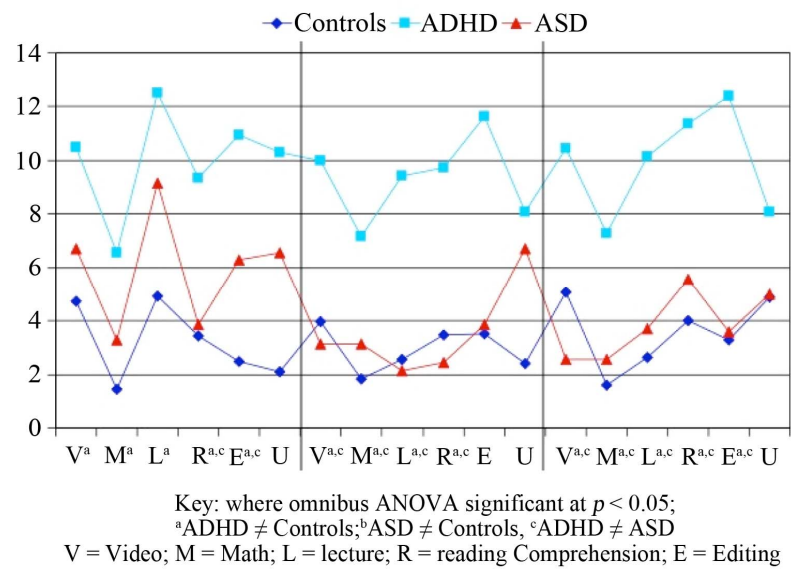

Figure 2. Self rating-inattention.

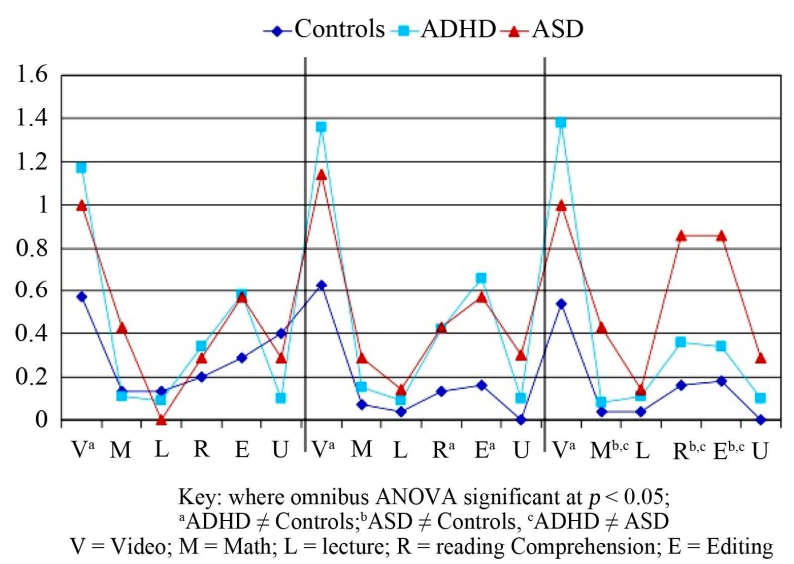

Figure 3. Self rating-hyperactivity.

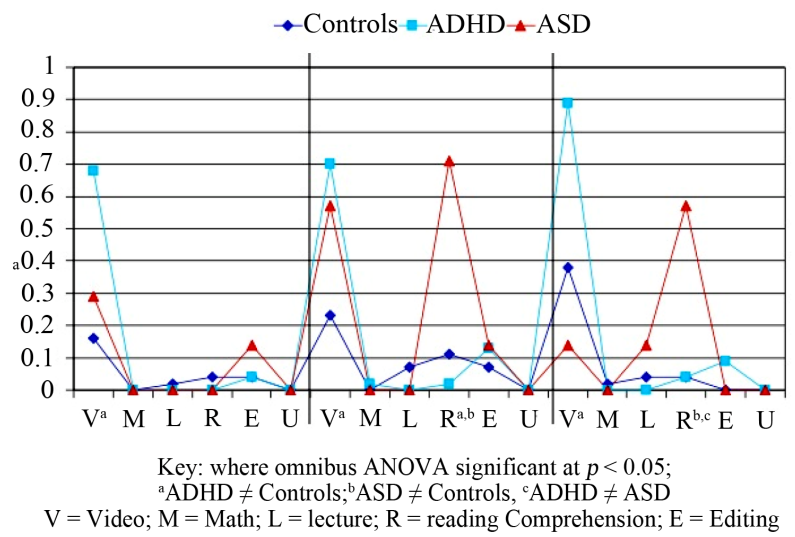

Figure 4. Self rating-inattention.

one-way ANOVAs and the associated post-hoc LSD tests are included in the figures. On self-report (Figures 1 and 2), there were no statistically significant differences between Controls and ASD. On the Observer reports differences, a small number of significant differences were found in the late afternoon (Figures 3 and 4 ).

As shown in Figure 5, there was a robust significant difference between the percent of tasks completed by the group with ASD (59.9\%) when compared both with Controls (82.7\%) and ADHD (82.6\%).

\section{DISCUSSION}

The main goal of this study was to examine workplace performance in adults with HF-ASD in a 10-hour simulated laboratory workplace using observer and self-reports. While no meaningful differences were observed in observer or self-ratings of inattention and hyperactivity, participants with HF-ASD were found to have significantly more difficulty in independent completion of unstructured tasks when compared to participants with ADHD and healthy Controls. Although in need of confirmation, these preliminary findings suggest that inde- 


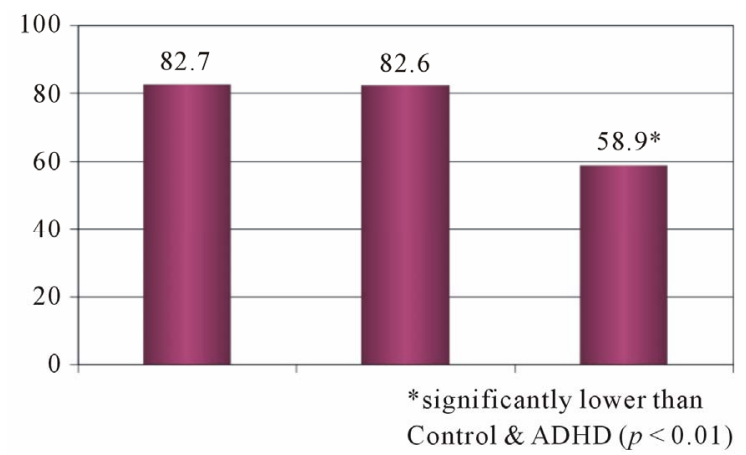

Figure 5. Precent of tasks completed.

pendent, unstructured work activities represent a key aspect of workplace deficits in individuals with HF-ASD that could be targeted for remediation.

Despite clear directions about which tasks were necessary to complete, individuals with HF-ASD completed only $58 \%$ of the assigned tasks, in comparison to an $82 \%$ completion rate by both the ADHD and Control groups ( $p=0.007)$. As most jobs require the ability to work independently, these findings shed light onto the difficulty of maintaining employment for individuals with HFASD. In fact, the Secretary of Labor's Commission on Achieving Necessary Skills (SCANS) [20] lists selfmanagement as a primary quality in being successful in the workplace. These findings stress the importance of highly structured tasks with immediate feedback loops to ensure workplace success for individuals with HFASDS.

As previously reported [13], ADHD participants reported statistically significant elevated inattentive and hyperactive symptoms throughout all three simulation periods. In contrast, HF-ASD subjects did not report subjective elevations of inattentive and hyperactive ADHD symptoms (Figures 1 and 2). Nonetheless, trained research observers rated HF-ASD participants to be significantly more hyperactive than ADHD participants and Controls in the late afternoon on the math, reading comprehension, and text editing tasks and significantly more inattentive than Controls during the early afternoon on the reading task (Figures 3 and $\mathbf{4}$ ). Contrary to our study hypothesis, participants with HF-ASD were 50\% less likely to report symptoms of hyperactivity or inattention than participants with ADHD observed in our previous work simulation study using the same paradigm [13].

While the findings that HF-ASD participants were less likely to report hyperactivity or inattention symptoms could reflect an absence of such symptoms, they alternatively may suggest a lack of self-awareness, a deficit frequently observed in treatment studies of individuals with ASD [21,22]. Self-awareness is critical for workplace success, as the differential from an employer's assessment of adequate workplace performance can be the deciding factor in keeping a job. Although the reasons for the absence of self awareness in this population are not fully understood, they may be due to the well documented deficits in theory of mind (TOM) associated with ASD [23], which can adversely impact awareness of self $[24,25]$, as well as awareness of others. Ponnett et al. underscored that structure is critical to individuals with ASD in being able to take another's perspective [26]. More work is needed to further examine the contributions of deficits in self-awareness and TOM on workplace performance of individuals with HF-ASD and to devise strategies to mitigate them. Future studies might also include more investigation of social interference as a variable that might adversely affect functioning and productivity.

Although few significant differences were found in specific task performances, HF-ASD participants exhibited a pattern of decline in performance of specific tasks in the later part of the work day. Likewise, observers noted more difficulties with attention and hyperactivity later in the day. These findings suggest that individuals with HF-ASD tend to disengage from optimal performance as the day progresses. Considering the importance of perseverance across the entire workday, this deficit could affect the ability of individuals with HF-ASD to sustain productivity in an actual job.

Our findings should be viewed in light of some methodological limitations. The sample size was small, limiting our ability to fully detect meaningful differences in all variables assessed. The laboratory workplace paradigm used was originally designed for adults with ADHD and not specifically for individuals with HF-ASD. Although we used the exact workplace paradigm, the assessment of individuals with HF-ASD was not done at the same time as those of ADHD and Controls.

Despite these limitations, this pilot study pioneered the use of a simulated workplace experience to safely and confidentially assess workplace skills and deficits in adults with HF-ASD. Our findings document that individuals with HF-ASD struggle with deficits with unstructured activities, that they may lack self-awareness, and that they struggle with declining performance in the later part of the workday. Considering that these deficits correspond well with SCANS's [20] report of skills needed for success in the workplace, if replicated in a larger study, our findings suggest that individuals with HF-ASD are at a high risk of being unsuccessful in the modern workplace without adequate accommodations. Given the fundamental importance of workplace success to adequate societal functioning, further study of this important issue is clearly warranted.

\section{REFERENCES}

[1] CDC (2006) Mental health in the united states: Parental report of diagnosed autism in children aged $4-17$ years 
—United states, 2003-2004. Morbidity and Mortality Weekly Report, 55, 481-486.

[2] Fombonne, E. (2003) The prevalence of autism. Journal of the American Medical Association, 289, 87-89. doi:10.1001/jama.289.1.87

[3] Baird, G., et al. (2006) Prevalence of disorders of the autism spectrum in a population cohort of children in south thames: The special needs and autism project (snap). Lancet, 368, 210-215. doi:10.1016/S0140-6736(06)69041-7

[4] Muller, E., Schuler, A. and Yates, G.B. (2008) Social challenges and supports from the perspective of individuals with asperger syndrome and other autism spectrum disabilities. Autism, 12, 173-190. doi:10.1177/1362361307086664

[5] Howlin, P., Alcock, J. and Burkin, C. (2005) An 8-year follow-up of a specialist supported employment service for high-ability adults with autism or asperger syndrome. Autism, 9, 533-549. doi:10.1177/1362361305057871

[6] Garcia-Villamisar, D. and Hughes, C. (2007) Supported employment improves cognitive performance in adults with autism. Journal of Intellectual Disability Research, 51, 142-150. doi:10.1111/j.1365-2788.2006.00854.x

[7] Higgins, K.K., Koch, L.C., Boughfman, E.M. and Vierstra, C. (2008) School-to-work transition and asperger syndrome. Work, 31, 291-298.

[8] Lawer, L., Brusilovskiy, E., Salzer, M.S. and Mandell, D.S. (2009) Use of vocational rehabilitative services among adults with autism. Journal of Autism and Developmental Disorders, 39, 487-494. doi:10.1007/s10803-008-0649-4

[9] Ganz, M.L. (2007) The lifetime distribution of the incremental societal costs of autism. Archives of Pediatrics and Adolescent Medicine, 161, 343-349. doi:10.1001/archpedi.161.4.343

[10] Meyer, R.N. (2001) Asperger syndrome employment workbook. Jessica Kingsley Publishers, London.

[11] Gaus, V. (2007) Cognitive-behavioral therapy for adult asperger syndrome. The Guilford Press, New York.

[12] Klin, A., Volkmar, F. and Sparrow, S. (2000) Asperger syndrome. Guilford Press, New York City.

[13] Fried, R., et al. (2012) A controlled study of a simulated workplace laboratory for adults with attention deficit hyperactivity disorder. Psychiatry Research, 200, 949-956. doi:10.1016/j.psychres.2012.04.020

[14] Joshi, G., et al. (2010) The heavy burden of psychiatric comorbidity in youth with autism spectrum disorders: A large comparative study of a psychiatrically referred population. Journal of Autism and Developmental Disorders, 40, 1361-1370. doi:10.1007/s10803-010-0996-9

[15] Joshi, G., et al. (2011) Discriminant and concurrent validity of a simplified DSM-based structured diagnostic instrument for the assessment of autism spectrum disorders in youth and young adults. BMC Psychiatry, 11, 204. doi:10.1186/1471-244X-11-204

[16] Wechsler, D. (1997) Wechsler adult intelligence scale III [manual]. The Psychological Corporation, San Antonio.

[17] Jastak, J. and Jastak, S. (1993) Wide range achievement test-third edition. Jastak Associates, Wilmington.

[18] Delis, D., Kaplan, E. and Kraemer, J.H. (2001) Deliskaplan executive function system (d-kefs). The Psychological Corporation, San Antonio.

[19] Robbins, T.W., et al. (1998) A study of performance on tests from the cantab battery sensitive to frontal lobe dysfunction in a large sample of normal volunteers: Implications for theories of executive functioning and cognitive aging. Journal of the International Neuropsychological Society, 4, 474-490. doi:10.1017/S1355617798455073

[20] Wise, L., Chia, W. and Rudner, L. (1990) American institutes for research the secretary's commission on achieving necessary skills. Pelavin Associates, Washington, DC.

[21] Thompson, T. (2008) Self-awareness: Behavior analysis and neuroscience. The Behavior Analyst, 31, 137-144.

[22] Verhoeven, E.W., et al. (2012) Brief report: Relationship between self-awareness of real-world behavior and treatment outcome in autism spectrum disorders. Journal of Autism and Developmental Disorders, 42, 889-894. doi:10.1007/s10803-011-1311-0

[23] Happe, F. (2003) Theory of mind and the self. Annals of the New York Academy of Sciences, 1001, 134-144.

[24] Jackson, P., Skirrow, P. and Hare, D.J. (2012) Asperger through the looking glass: An exploratory study of self-understanding in people with asperger's syndrome. Journal of Autism and Developmental Disorders, 42, 697-706.

[25] Mundy, P., Gwaltney, M. and Henderson, H. (2010) Selfreferenced processing, neurodevelopment and joint attention in autism. Autism, 14, 408-429.

[26] Ponnet, K., Buysse, A., Roeyers, H. and De Clercq, A. (2008) Mind-reading in young adults with asd: Does structure matter? Journal of Autism and Developmental Disorders, 38, 905-918. 